\author{
FRANCEMED \\ (Daniel KöNIG, Yassir BenHIMA, Rania ABDELlatif, Elisabeth RuCHAUd)
}

\title{
Les acteurs des transferts culturels Introduction à la thématique
}

\begin{abstract}
AVANT-PROPOS
Le présent volume est le produit d'un colloque organisé à l'Institut historique allemand, Paris, du 20 au 22 janvier 2010, par le groupe de recherche FranceMed (»La France et la Méditerranée. Espaces des transferts culturels«). Intitulé »Les acteurs des transferts culturels en Méditerranée médiévale. Sphères d'activité, contributions, fonctions«, ce colloque faisait partie d'un cycle de conférences sur »Les transferts culturels en Méditerranée médiévale« composé de quatre manifestations qui portaient sur (1) la construction historiographique de la Méditerranée, (2) les acteurs des transferts culturels, (3) le rôle des artefacts et des objets d'art dans les transferts culturels ainsi que (4) les différentes formes de mobilité et leur reconstruction' 1 .

La mise en œuvre et le bon déroulement de ce cycle de conférences, mais aussi la publication des actes du premier et du deuxième colloque ${ }^{2}$, n'auraient pas été possibles sans le soutien financier de la Deutsche Forschungsgemeinschaft (DFG) et de l'Institut historique allemand, Paris. Ces conférences ont bénéficié de l'appui personnel de plusieurs membres du conseil scientifique de l'institut, en l'occurrence des professeurs Martin Kintzinger, Claudia Zey et Bernd Schneidmüller, mais aussi de plusieurs membres de l'institut même, notamment sa directrice, Mme le professeur Gudrun
\end{abstract}

1 Premier colloque du 9-10 juin 2009: »Construire la Méditerranée, penser les transferts culturels. Approches historiographiques et perspectives de recherche«, compte-rendu en anglais: H-Soz-u-Kult, [25/7/2009], http://hsozkult.geschichte.hu-berlin.de/tagungsberichte/id=2708 (18/11/2011); deuxième colloque du 20-22 janvier 2010: »Les acteurs des transferts culturels en Méditerranée médiévale. Sphères d'activité, contributions, fonctions«, compte-rendu en anglais: H-Soz-u-Kult, [18/3/2010], http://hsozkult.geschichte.hu-berlin.de/tagungsberichte/id= 3032 (18/11/2011); troisième colloque du 24-25 mars 2010: »Objets et art dans les transferts culturels«, compte-rendu anglais: H-Soz-u-Kult, [19/5/2010], http://hsozkult.geschichte.huberlin.de/tagungsberichte/id=3117; quatrième colloque du 1-2 juin 2010: »Mobilité(s). Pour une étude des sources et des méthodes d'approche«, compte-rendu en anglais: H-Soz-u-Kult, [2/7/2010], http://hsozkult.geschichte.hu-berlin.de/tagungsberichte/id=3174 (18/11/2011). Une dernière manifestation a été organisée, les 9-11 mars 2011, en coopération avec la Casa de Velázquez (Daniel Baloup) sous le titre »Itinérances des savoirs et des biens culturels. Pour une analyse spatiale des transferts culturels en Méditerranée médiévale«, compte-rendu en anglais: H-Soz-u-Kult, [10/4/2011], http://hsozkult.geschichte.hu-berlin.de/tagungsberichte/id=3604 $(18 / 11 / 2011)$.

2 Voir la publication des actes du premier colloque: Rania ABDELlatiF, Yassir BenHIMA, Daniel KÖNIG, Elisabeth RUCHAUD (dir.), Construire la Méditerranée, penser les transferts culturels. Approches historiographiques et perspectives de recherche, Munich 2012. 
Gersmann, le coordinateur scientifique, Stephan Geifes, la responsable des manifestations scientifiques, Dunja Houelleu, les membres de l'administration Ralf Nädele, Sara Windgassen et Ulrika Saga, les agents techniques et de l'accueil Roger Klimke, Sabrina Mengeler et Britta Oleinek ainsi que le service des publications, représenté par Veronika Vollmer et Claudie Paye et soutenu par les stagiaires Perrine Thierrée, Anne-Lise Péco et Katharina Maginot. Nous leur exprimons nos remerciements les plus profonds pour avoir soutenu nos activités dans un esprit de coopération fructueuse.

\section{L'ACTEUR, SUJET DE L'HISTOIRE}

Tout processus d'échange entre différentes aires culturelles, quel que soit l'outil terminologique utilisé pour en décrire le déroulement et les effets ${ }^{3}$, s'effectue sur la base de quatre éléments fondamentaux. Il s'agit (1) des objets, matériels ou immatériels, mutables ou immutables, vivants ou inanimés qui subissent ces processus; (2) des structures qu'on pourrait qualifier de `spatiales〈, qui conditionnent le cadre matériel, le support de mobilité, les réseaux humains et les orientations symboliques nécessaires pour faciliter la mobilité géographique de ces objets; (3) des lieux, au sens le plus large, de mémoire et de conservation qui permettent la durabilité et, par conséquent, la >mobilité temporelle` des objets d'une grande longévité; (4) enfin les acteurs qui servent de stimulants ainsi que d'exécuteurs et qui se distinguent les uns des autres par leur motivation, leur capacité et leur degré d'implication dans ces processus 4 .

L'acteur humain a toujours été au centre de la production historiographique. Les peintures et gravures de l'art pariétal, les inscriptions commémoratives immortalisant des batailles victorieuses, l'éloge funèbre gravé sur une épitaphe, mais aussi des formes littéraires plus élaborées, notamment le panégyrique, les gesta, la biographie et les recueils biographiques ainsi que, finalement, l'autobiographie, témoignent tous du même besoin humain de se présenter, de décrire, mais aussi de juger les activités de son espèce. La recherche historique, centrée sur le genre humain, mettant en exergue son activisme et excluant souvent l'apport des facteurs non humains dans le déroulement des processus historiques ${ }^{5}$, suit le même cheminement, bien qu'elle utilise plusieurs genres qui ne sont pas nécessairement >biographiques<. Nombreuses sont les publications qui comportent le mot »acteur« dans leur titre ${ }^{6}$. Et malgré le fait que

3 Voir la discussion de ces outils terminologiques, notamment les notions d'»acculturation «, de »métissage«, de »traduction« et de »transfert culturel« dans: FRANCEMED (Rania ABDELLATIF, Yassir BENHIMA, Daniel KÖNIG, Elisabeth RUCHAUD), Introduction à l'étude des transferts culturels en Méditerranée médiévale, dans: ID. (dir.), Construire la Méditerranée (voir n. 2), sous-partie »Histoire d'une notion: aux origines du transfert culturel «.

4 Ibid., sous-partie »Les éléments fondamentaux des transferts culturels «.

5 Il y a, bien sûr, des contre-exemples, comme la monographie de William MCNEILL, Plagues and Peoples, Garden City 1976, qui traite de l'influence des bactéries et des parasites sur l'évolution de l'histoire humaine.

6 À titre d'exemple: Sylvie Humbert, Vincent Bernaudeau (dir.), Auteurs et acteurs de la séparation des Églises et de l'État, Lille 2007. 
l'éthique professionnelle empêche l'historien de glorifier les actes d'un être humain, la recherche historique contribue à jamais à sauvegarder, à pérenniser et, par conséquent, à surdimensionner l'importance de cet acteur qu'est l'homme et qui, vu de la perspective du temps géologique et de l'histoire écologique, n’est qu'un >parvenu< récent, amené à disparaître?

Les historiens ont abordé le sujet des >acteurs` sous divers angles, dont la variété mérite d'être mentionnée, sans prétendre que les exemples suivants soient représentatifs d'une quelconque origine nationale, ethnique, religieuse ou intellectuelle. La survalorisation, par la glorification, du rôle de l'acteur en histoire, illustrée, à titre d'exemple, par la monographie de 'Abd Allāh Nāṣiḥ 'Ulwān intitulée »Saladin l'Ayyubide, héros de Hatțīn et libérateur de Jérusalem $\aleph^{8}$, a trouvé une forme quasiment étatique dans les pays socialistes ou communistes comme la RDA, dont la production historiographique est souvent caractérisée par la nécessité idéologique de mettre la classe ouvrière ou le prolétariat au centre de l'enquête scientifique9. Une telle surévaluation de l'être humain a déjà été analysée en tant que phénomène historique par Thomas Carlyle en 1840 dans son livre »On Heroes, Hero-worship and the Heroic in History «10. Par ailleurs, elle constitue la thématique principale de plus d'une publication sur l'héroïque comme phénomène de la production littéraire des sociétés du passé11.

Mais cette célébration du héros est aujourd'hui largement abandonnée par une pratique historienne moderne rétive aux usages de l'histoire plutôt nationaliste d'antan. Cela n'empêche pas un engouement sans précédent pour les biographies historiques, d'où certains grands succès en librairie. Malgré les contraintes qu'implique l'écriture pour le grand public, le souci d'objectivité marque désormais toute approche du genre biographique $^{12}$. La biographie scientifique, produite en masse, notamment sur des

7 Voir Jens HARDER, Alpha ...directions, Arles 2009, pour un aperçu artistique bien documenté de l'histoire préhumaine. Voir également Dougal DixON, John ADAMs, The Future is Wild, Oxford 2004, pour une vision du futur de la planète - sans êtres humains!

8 'Abd Allāh Nāṣiḥ 'UlwĀN, Șalāḥ ad-Dīn al-Ayyūbī: baț̣al al-Ḥațīin wa muharrir al-Quds min aș-șalībiyyīn, Beyrouth 1983.

9 Voir Alf LÜDTKE, Wer handelt? Die Akteure der Geschichte. Zur DDR-Geschichtsschreibung über Arbeiterklasse und Faschismus, in: Georg G. Iggers, Die DDR-Geschichtswissenschaft als Forschungsproblem, Munich 1998, p. 369-410 (Beihefte der Historischen Zeitschrift, 27). Que l'historiographie socialiste et communiste est quand même beaucoup plus nuancée et a contribué beaucoup à la mise en valeur des facteurs économiques se note en lisant Alexander DEMANDT, Der Fall Roms. Die Auflösung des römischen Reiches im Urteil der Nachwelt, Munich 1984, p. 316-346, 419-426, sur l'interprétation de la chute de l'Empire romain selon l'interprétation des pays du bloc communiste.

10 Thomas CARlyle, On Heroes, Hero-worship and the Heroic in History, Londres 1840; voir aussi Benjamin H. LeHMAN, Carlyle's Theory of the Hero. Its Sources, Development, History, and Influence on Carlyle's Work, Durham 1928.

11 Voir p.ex. Şevket KÜÇÜKHÜSEYIN, Die Rolle von Gewalt bei der Konstruktion exemplarischer Persönlichkeitsideale, in: Michael BORGOLTE, Juliane SCHIEL, Bernd SCHNEIDMÜLLER, Annette SEITZ (dir.), Mittelalter im Labor. Die Mediävistik testet Wege zu einer transkulturellen Europawissenschaft, Berlin 2008, p. 466-495, avec plus de références bibliographiques et beaucoup d'exemples tirés de l'époque médiévale.

12 Sur l'engouement pour les biographies historiques et les caractéristiques du genre, François Dosse, Le pari biographique. Écrire une vie, Paris 2005. 
personnes considérées comme des figures >fondatrices`d'un groupe ou d'une société spécifiques ou responsables d'une évolution déterminante (comme Charlemagne, Napoléon ou encore Marx) ne peut toujours échapper à la subjectivité, vu que le processus de l'écriture biographique entraîne souvent une identification, ou du moins une certaine empathie avec le sujet ${ }^{13}$.

Surtout quand l'acteur et le témoin ne font qu'un, la mise en récit du fait historique est forcément teintée de subjectivité. Il en est de même pour le témoignage autobiographique. Mais dans l'optique de reconstituer le rôle des acteurs de l'histoire, l'autobiographie peut s'avérer d'un apport singulier. L'historien peut y trouver, en plus de l'autoreprésentation littéraire, l'expression des tréfonds d'une personnalité, avec ses émotions et ses motivations ${ }^{14}$.

Loin du culte du héros, les biographies collectives sanctionnent une volonté séculaire de placer l'individu dans le moule du groupe ou de la société. Plus centrées sur des données censées être plus >objectives`, les recueils de biographies s'avèrent plus compatibles avec l'esprit et les pratiques de l'histoire moderne. Les méthodes de la prosopographie scientifique, particulièrement aidées par les possibilités nouvelles qu'offre l'outil informatique, autorisent la sociographie de groupes divers grâce à l'exploitation quantitative et qualitative des données sur les biographies et les réseaux sociaux des individus concernés ${ }^{15}$. En contribuant à cerner les identités collectives des (ou de certains) acteurs de l'histoire, la prosopographie prolonge en une certaine manière les fins des auteurs du passé. Qu'il s'agisse des catalogues d'écrivains de l'Antiquité tardive (comme celui écrit par Jérôme à la fin du IV et complété par Gennadius de Marseille à la fin $\mathrm{du} \mathrm{v}^{\mathrm{e}}$ siècle $^{16}$ ), ou des tabaqāt, recueils biobibliographiques des savants arabo-musulmans de la période classique ${ }^{17}$, écrire des biographies collectives participe d'une stratégie de distinction qui vise à la reproduction sociale d'un groupe circonscrit.

Beaucoup de questions restent encore ouvertes, notamment les questions philosophiques liées au phénomène de l'acteur et son interprétation historique. Comment, par exemple, harmoniser la notion du libre arbitre avec la causalité compulsive, presque

13 Sur le développement de ce genre: Olaf HÄHNER, Historische Biographik. Die Entwicklung einer geschichtswissenschaftlichen Darstellungsform von der Antike bis ins 20. Jahrhundert, Francfort/M. 1999.

14 Voir surtout Georg MISCH, Geschichte der Autobiographie, 4 vol., Leipzig, Berlin 1907-1969; voir aussi Günter NIGGL (dir.), Die Autobiographie. Zu Form und Geschichte einer literarischen Gattung, Darmstadt ${ }^{2}$ 1998. Pour le monde arabo-musulman, Dwight F. REYNOLDS (dir.), Interpreting the Self. Autobiography in the Arabic Literary Tradition, Los Angeles 2001.

15 Voir p.ex. Averil CAMERON (dir.), Fifty Years of Prosopography: The Later Roman Empire, Byzantium and Beyond, Oxford 2003; Konrad Vössing, Einleitung, dans: ID. (dir.), Biographie und Prosopographie, Stuttgart 2005, p. 9-10. Pour le monde musulman médiéval, soulignons la contribution fondamentale de la série Estudios onomástico-biográficos de al-Andalus (15 volumes parus à ce jour).

16 Hieronymus et Gennadius Massiliensis, De viris inlustribus, ed. Carl Albrecht Bernoulli, Freiburg, Leipzig, 1895, réimpression Francfort/M. 1968.

17 Voir Claude GiLliot, Tabakāāt, dans: Encyclopaedia of Islam 2, Leyde 2000, vol. 10, p. 7; Paul AuChTERlonie, Arabic Biographical Dictionaries. A Summary Guide and Bibliography, Durham 1987. 
obsessionnelle, d'une recherche historique qui a, la plupart du temps, délégué la responsabilité de traiter ce lien complexe entre volonté et causalité à un champ de recherche dénommé »philosophie de l'histoire«18? Certains représentants de la philosophie contemporaine commencent et concluent leur raisonnement avec la phrase »Free will remains a mystery ${ }^{19}{ }^{9}$, remettant en question les compromis traditionnels de nature religieuse. Par conséquent, la question du libre arbitre pose encore des problèmes pour toute analyse historique qui tente de définir la marge de manœuvre de l'être humain au sein des grands processus historiques.

Prenons l'exemple de la christianisation de l'Europe occidentale du $\mathrm{IV}^{\mathrm{e}}$ au VIII ${ }^{\mathrm{e}}$ siècle. La question du rapport entre »conversion et liberté«, thématique principale d'une monographie excellente de Bruno Dumézil, est toujours à considérer dans ce champ de recherche ${ }^{20}$. Cependant, les facteurs et contraintes qui contribuent à imposer le système chrétien aux sociétés romaines et postromaines de l'Europe occidentale ne sont pas tous créés par des élites puissantes et soucieuses de sauvegarder ou d'amplifier leur pouvoir. Évidemment, ces élites contribuent à la diffusion de ce système. Mais elles sont, elles aussi, le produit des processus au sein desquels elles agissent, des processus historiques qui sont trop complexes pour être du ressort d'individus ou de groupes. Quel choix avait un individu quelconque, éduqué d'une certaine manière, marqué par certaines expériences et poussé par certains facteurs, s'il avait 〉choisiく de se convertir par >conviction`, pour éviter une situation de pression ou pour s'en libérer, ou même pour profiter, d'une manière ou d'une autre, de sa conversion'21? Au-delà de la marge étroite de manœuvre accordée à l'homme par les circonstances de son existence, les méthodes de recherche historique semblent renforcer l'impression que l'action de l'homme est délimitée par des facteurs sociaux et culturels indépendants de sa propre volonté22. Reprenant l'exemple du converti au christianisme, quelle liberté de choix lui accorde-t-on en tant qu'historien, forcé de reconstruire une telle évolution dite religieuse à la base d'une logique causale qui explique toute action et toute décision humaine en considérant et reconstruisant les facteurs qui les incitent ${ }^{23}$ ? La question reste, donc: qui est un >vrai acteur/, c'est-à-dire une puissance capable de mettre en marche des processus historiques sans être uniquement l'annexe causale des puissances loin-

18 Voir, p.ex., les réflexions de: Edward Hallett CARR, What is History?, Londres 1962, p. 88, 90, 102; Karl ACHAM, Analytische Geschichtsphilosophie. Eine kritische Einführung, Freiburg 1974, p. 133, 190-191, 329-335; Maurice MANDELBAuM, Anatomy of Historical Knowledge, Baltimore, Londres 1977, p. 105-111, 138; Mario Augusto BuNGE, Kausalität, Geschichte und Probleme, Tübingen 1987, p. 118-119, 296-299, 304.

19 Peter van InWagen, Free Will Remains a Mystery, dans: Philosophical Perspectives 12 (2000), p. 1-19.

20 Bruno DumÉZIL, Les racines chrétiennes de l'Europe. Conversion et liberté dans les royaumes barbares ( $\mathrm{V}^{\mathrm{e}}$ au VIII ${ }^{\mathrm{e}}$ siècle), Paris 2006.

${ }^{21} \mathrm{Ce}$ sont les trois grandes catégories de motivations qui figurent dans Daniel KöNIG, Bekehrungsmotive. Untersuchungen zum Christianisierungsprozess im römischen Westreich und seinen romanisch-germanischen Nachfolgern (4.-8. Jahrhundert), Husum 2008.

22 On peut voir aussi, p.ex., Mohamed HADI CHERIF, Abdelhamid HÉNIA (dir.), Individu et pouvoir dans les pays islamo-méditerranéens, Paris 2009.

23 KÖNIG, Bekehrungsmotive (voir n. 21), p. 12-14. 
taines? Si l'homme n'était qu'une annexe, on pourrait lui accorder le même statut d'acteur que celui qu'accorde aux images Michel Poivert, le directeur d'un livre intitulé »Événements. Les images comme acteurs de l'histoire«24.

Le présent volume ne veut et ne peut pas répondre aux questions de ce genre. Mais il cherche malgré tout à fournir un aperçu, incomplet, du rôle et des formes d'implication d'acteurs divers dans le contexte multiculturel de la Méditerranée entre l'Antiquité tardive et le bas Moyen Âge. Comment rendre compte de l'implication de cette multiplicité d'acteurs dans les processus complexes de transferts culturels en Méditerranée médiévale? Comment éviter les écueils d'une survalorisation du rôle de certains individus et groupes dans cette dynamique de transferts? Les différentes contributions à ce volume répondent en filigrane à ces deux questionnements, dont la portée historique dépasse la simple curiosité académique pour embrasser des préoccupations plus contemporaines. En effet, plusieurs des sujets abordés pour la Méditerranée médiévale font écho aux situations de contacts intra-, inter- ou transculturels dans les sociétés contemporaines, notamment en Europe occidentale, où les questions liées à la place des populations immigrées et au rôle de l'islam se posent avec acuité, et parfois en termes polémiques.

\section{ACTEURS ET CONTEXTES}

Mais le but de ce livre n'est pas nécessairement de mettre en évidence la contribution potentielle de ceux qui circulent ou qui se trouvent >entre < les cultures. Il s'agit plutôt d'analyser un des phénomènes fondamentaux de chaque processus d'échange et de transfert qui est son stimulant, son moteur, bref, son acteur. Réduire les échanges et transferts culturels aux mécanismes et suggérer qu'ils se déroulent d'une façon quasiment automatique dans toute situation de rencontre entre différents systèmes culturels néglige le poids des conditions sociopolitiques et socioculturelles qui constituent le cadre dans lequel agit un acteur quelconque. Bien qu'il y ait aussi des acteurs qui agissent indépendamment des systèmes de normes et de valeurs de leur société - certaines activités commerciales des cités-États maritimes italiennes avec le monde musulman en dépit des interdictions de l'Église en fournissent un bel exemple ${ }^{25}$ - il est évident que ces conditions sociopolitiques et socioculturelles peuvent aussi bien renforcer des fragmentations et confrontations identitaires que faciliter la création des réseaux inter- et transculturels qui constituent la base des échanges et des transferts.

À la diversité des acteurs, de leur position et degrés d'implication, correspondent donc des environnements socioculturels et politiques très variables. L'enfant dont les parents appartiennent à des groupes religieux, ethniques et/ou linguistiques différents a

24 Michel POIVERT (dir.), Événements. Les images comme acteurs de l'histoire, Paris 2007.

25 Voir p.ex. les stipulations du quatrième concile du Latran: Concilium Lateranense IV (1215),

$\S 71$, dans: Joseph WoHLmuTH (ed.): Konzilien des Mittelalters. Vom ersten Laterankonzil (1123) bis zum fünften Laterankonzil (1512-1517), Paderborn 2000, p. 270,2-9, ainsi que la critique dirigée contre les cités-États par Guillelmus Adae, De modo Sarracenos extirpandi (écrit après 1317), dans: Recueil d'histoire des croisades. Documents arméniens, vol. 2, p. 523. 
évidemment une position qui se distingue nettement de celle de l'émissaire politique ou du missionnaire incapables d'opérer sans interprète ${ }^{26}$. Un traitement exhaustif de cette diversité n'est pas possible; et les 17 contributions de ce volume, malgré leurs champs de spécialisation variés, ne peuvent en aucun cas y prétendre. Ces textes, organisés en trois sections complémentaires, représentent une partie des acteurs du contact interculturel en Méditerranée médiévale, mais laissent aussi apparaître des lacunes inhérentes à tout travail, forcément incomplet, sur un sujet aussi vaste.

La première section, »Acteurs en mouvement«, est composée d'articles qui mettent en scène des acteurs dont la mobilité géographique est déterminante dans la circulation d'idées et de biens culturels. Plusieurs exemples illustrent des formes et des motivations diverses de mobilité. Les pèlerins chrétiens vers Jérusalem, étudiés ici par Elisabeth Ruchaud, sont mus par une quête spirituelle qui les entraîne sur les chemins d'un Orient représenté entre le vécu et le fantasmé. L'implication des ambassadeurs dans les transferts culturels depuis ou vers Byzance est traitée par la contribution de Nicolas Drocourt. Michel Balard traite du rôle de passeurs culturels des migrants génois vers l'outre-mer. Dans ces articles, les transferts culturels se déroulent grâce à, et en conséquence de cette mobilité. Évidemment, beaucoup de types d'acteurs agissant grâce à leur mobilité ne sont pas traités dans cette section. Les grands mouvements migratoires autour et au sein de la Méditerranée médiévale sont absents de ce volume. De par leur ampleur démographique et leur étendue géographique, plusieurs mouvements migratoires ont contribué d'une manière décisive à la circulation des idées et des biens culturels. C'est le cas des migrations des Visigoths, des Vandales et d'autres groupes qui engendrèrent la création des royaumes dits >germaniques` autour de la Méditerranée occidentale, mais aussi de l'expansion arabo-musulmane qui entraîna la création des sociétés gouvernées par des élites musulmanes entre al-Andalus et l'Asie centrale. L'arrivée des Bulgares et des Hongrois dans les Balkans, l'installation des Normands en Italie méridionale, le mouvement des Banū Hilāl et Banū Sulaym depuis l'Égypte vers le Maghreb ainsi que l'occupation de l'Anatolie par les Seljouks et d'autres groupes dits >turcs` en Anatolie ne représentent qu'une partie des mouvements migratoires dont l'impact sur la Méditerranée et ses environs se ressent jusqu'à nos jours. Les mouvements d'expansion politique, militaire, mais aussi commerciale à l'instar des (re-)conquêtes de Justinien, de l'expansion fatimide depuis le Maghreb jusqu'en Syrie, les conquêtes almoravides et almohades ainsi que la prétendue reconquête espagnole - sans oublier les croisades et l'essor économique des cités-États italiennes sont aussi liés à une mobilité massive des personnes. Par conséquent, les conquérants, gouvernants et administrateurs, les réfugiés, mais aussi les marchands, les marins, les esclaves, etc. figurent nécessairement parmi les groupes dont la mobilité à travers l'espace méditerranéen médiéval mérite d'être évoquée.

26 Voir une analyse systématique de ces points dans: FRANCEMED, Introduction à l'étude des transferts culturels (voir n. 3), sous-partie »Objets - espaces - acteurs«. 
Les articles de la deuxième section, intitulée »Hommes de l'entre-deux « d'après le titre d'un ouvrage récent de Bernard Heyberger et de Chantal Donzel-Verdeil27, se regroupent autour des acteurs qui se trouvent soit au entre plusieurs groupes, soit entre plusieurs milieux socioculturels: l'article de Daniel König est consacré au phénomène des personnalités bi- ou multiculturelles en analysant leur position - précaire et instable, mais aussi profitable et avantageuse selon les circonstances - entre différentes sphères linguistiques, religieuses, sociales et culturelles. Ce genre de personnalités joue un grand rôle aussi dans d'autres contributions: les problématiques causées par la présence des otages au centre d'al-Andalus umayyade sont au centre de la contribution de Maribel Fierro. Juda Halévi, célèbre savant juif d'al-Andalus, se retrouve dans sa pratique du voyage à la croisée de deux cultures littéraires, hébraïque et arabomusulmane, comme le démontre l'analyse de Yann Dejugnat. Filippo Malerbi, le »spécialiste du transfert clandestin « étudié par Georg Christ, fournit l'exemple d'un indivi$\mathrm{du}$ qui franchit régulièrement les limites sociales entre la diaspora vénitienne en Égypte et son environnement mamelouk. Finalement, Johannes Pahlitzsch nous introduit non seulement aux relations entre les Mamelouks et Chypre, mais encore à la vie familiale d'un souverain mamelouk qui est caractérisée par des liens transculturels divers. D'autres études de cas pourraient suivre pour souligner l'importance de ce genre d'hommes qui témoignent de l'existence des >espaces tiers`, de >métissageく ou des formes d'’hybridité<, c'est-à-dire de zones transitoires entre aires culturelles, mais aussi des frontières et limites imposées par des circonstances socioculturelles variées. La figure la plus emblématique de ces espaces transitoires n'a pas été traitée en détail dans ce cadre malgré son importance pour tout transfert et échange de nature linguistique: le traducteur et/ou interprète. Évoqué dans plusieurs contributions de ce volume ${ }^{28}$, cette figure - impliquée dans la traduction des œuvres scientifiques entre les langues écrites de la Méditerranée médiévale et de ses périphéries, dans les négociations politiques et commerciales ainsi que dans la formulation et traduction des traités - aurait mérité d'être représentée dans ce volume ${ }^{29}$.

Plusieurs articles, regroupés dans la troisième section, intitulée »Les acteurs et leurs empreintes«, approchent la thématique principale en reconstruisant les acteurs autour d'un processus de transfert spécifique: Jean-Charles Ducène analyse et interprète les sources de la connaissance de l'Europe chez les géographes arabo-musulmans en évoquant des acteurs qui contribuent à la formation et l'évolution des images de l'»autre«. Se basant sur les portulans, Sonja Brentjes s'interroge sur les personnes impliquées dans la transmission et la réception des savoirs nécessaires pour leur pro-

27 Bernard Heyberger, Chantal Donzel-Verdeil (dir.), Hommes de l'entre-deux. Parcours individuels et portraits de groupes sur la frontière de la Méditerranée $\left(\mathrm{XVI}^{\mathrm{e}}-\mathrm{XX}^{\mathrm{e}}\right.$ siècle), Paris 2009.

28 Voir surtout les contributions de Raphaela Veit et de Regula Forster.

29 À propos de cette dernière perspective, soulignons l'intérêt de la publication de María Teresa Ferrer Mallol, Jean-Marie Moeglin, Stéphane PÉQuignot et al. (dir.), Negociar en la Edad Media. Négocier au Moyen âge, Barcelone 2005. Sur cette thématique, voir aussi le numéro 8 (2011) du journal »Trivium. Revue franco-allemande de sciences humaines et sociales«, http://trivium.revues.org/3868 (18/11/2011). 
duction. Raphaela Veit et Juliette Sibon se focalisent sur le transfert de pratiques et de savoirs médicaux: tandis que la première traite la transmission, traduction et réception des textes médicaux en Italie médiévale, la deuxième se penche sur les échanges professionnels entre médecins juifs et chrétiens à Marseille. Les domaines technique et architectural sont au centre d'une contribution de Rania Abdellatif qui reconstruit l'éventail des acteurs impliqués dans la transformation des bâtiments religieux en Orient de l'époque ayyubide, sur la base des textes, mais aussi de l'étude architecturale des monuments. En traitant la diffusion et la transformation d'un sujet littéraire de l'Antiquité jusqu'au XVII ${ }^{\mathrm{e}}$ siècle, Regula Forster montre qu'une analyse textuelle permet de reconstruire l'activité d'un grand nombre d'acteurs dont l'identité et la biographie resteront presque toujours cachées. Enfin, dans une approche plutôt macrohistorique et comparatiste, Georg Jostkleigrewe relève le défi de définir la spolitique étrangère` d'un royaume de la période préétatique, en soulignant les spécificités d'une époque et de ses acteurs et décideurs politiques.

Les mots de clôture sont de Pierre Guichard et prennent la forme d'une conclusion qui ne se contente pas de passer en revue les thématiques de ce volume, mais qui crée un lien avec les discussions actuelles autour des relations entre mondes arabomusulman et latino-chrétien, du point de vue d'un chercheur reconnu et chevronné. 
